\title{
What are the necessary corrections for dynamic cardiac SPECT?
}

\author{
Brian F. Hutton, PhD, ${ }^{\mathrm{a}, \mathrm{b}}$ and Simona Ben-Haim, MD, DSc ${ }^{\mathrm{a}, \mathrm{c}}$ \\ a Institute of Nuclear Medicine, University College London and UCL Hospitals, London, United \\ Kingdom \\ ${ }^{b}$ Centre for Medical Radiation Physics, University of Wollongong, NSW, Australia \\ c Institute of Nuclear Medicine, Chaim Sheba Medical Center, Tel-Hashomer, Israel
}

Received May 22, 2016; accepted May 23, 2016

doi: 10.1007/s12350-016-0580-6

See related article, pp. 1332-1346

\section{WHAT ARE THE NECESSARY CORRECTIONS FOR DYNAMIC CARDIAC SPECT?}

Myocardial blood flow (MBF) and myocardial flow reserve (MFR) are important physiologic parameters for the detection of hemodynamically significant coronary artery disease (CAD) and have been shown to improve diagnostic accuracy and risk stratification of myocardial perfusion imaging (MPI), beyond that provided by relative perfusion abnormalities alone. Quantitative assessment of MBF can be obtained from cardiac PET, however, it is infrequently used in clinical practice due to the limited availability of PET scanners, suitable radiotracers, and dedicated software as compared to the widely used SPECT. MPI SPECT with conventional Anger technology has been traditionally limited to visual analysis or semiquantitative perfusion analysis. SPECT quantification of MBF requires fast acquisition of dynamic data in 5-10 seconds, as well as corrections, mainly for attenuation and scatter, which enable absolute measurement.

Wang et al. have assessed dynamic SPECT on a conventional SPECT scanner with NaI detectors. CT was acquired separately using a low-radiation dose protocol and was used for attenuation correction. ${ }^{1}$ The authors demonstrate that estimation of MBF requires rigorous correction of factors that affect quantification,

Reprint requests: Simona Ben-Haim, MD, DSc, Institute of Nuclear Medicine, Chaim Sheba Medical Center, Tel-Hashomer, Israel; simona.ben-haim@sheba.health.gov.il

J Nucl Cardiol 2017;24:1347-9.

$1071-3581 / \$ 34.00$

Copyright (C) 2016 American Society of Nuclear Cardiology. including attenuation and scatter. In general, the nuclear cardiology community has been reluctant to embrace the recommendations of the Society of Nuclear Medicine and American Society of Nuclear Cardiology ${ }^{2}$ regarding attenuation correction for myocardial SPECT with most clinical sites preferring to rely on clinical interpretation by experienced practitioners. Direct measurement of attenuation without a CT system is difficult, if not impossible, for certain acquisition geometries, and there is growing concern regarding the additional radiation dose that results from cardiac CT protocols. Clearly without attenuation correction, addressing additional sources of quantification error is futile. The authors illustrate that accounting for additional factors does result in more reliable quantitative results with the addition of scatter correction and resolution modeling in addition to solely attenuation correction, provided that noise is adequately controlled. Missing from their analysis was correction for attenuation alone, which most likely accounts for the largest quantitative error. Scatter accounts for a sizable fraction of photopeak events in conventional SPECT systems. Although this is reduced in cadmium zinc telluride (CZT) systems due to their superior energy resolution, appropriate correction is still needed in conjunction with attenuation correction. Resolution modeling helps to minimize the spatial variation of reconstructed resolution, but does not fully correct for the partial volume effects that influence accuracy of measurement. In general, from the perspective of both quality and quantification, the more exact the system model, the better the reconstruction.

Partial volume effects due to the limited resolution of SPECT will introduce a bias in estimation of parameters for objects that approach the resolution of the system. Myocardial thickness is of the same order as the typical reconstructed resolution and is subject to partial volume effects, as it is obvious from the brightening appearance due to wall thickening during 
contraction. As stated above, modeling resolution in the reconstruction will attempt to recover contrast, however, this will not fully correct for the partial volume effects. There are a large number of partial volume correction methods available ${ }^{3}$, but often these tend to rely on availability of anatomical imaging from which the myocardium can be segmented and few methods, if any, are available for routine clinical use, especially for the heart. Full quantification does require this additional correction if bias is to be avoided.

A factor that the authors did not address is motion, due to both cardiac contraction and respiration. Both result in blurring of the myocardial wall, with respiration mainly blurring in the axial direction. The effect on quantification will depend on the degree of motion, influenced by ventricular function and breathing pattern, both potentially different in rest and stress conditions. ${ }^{4}$ Linked to this is the potential mismatch between emission and transmission (CT) studies which may also differ in the two conditions. Accounting for motion is particularly challenging in kinetic studies ${ }^{5}$ where the count level in a single dynamic frame is already compromised, so ECG or respiratory gating of the data potentially results in unacceptably poor statistics. Respiratory motion correction strategies are often applied in PET studies as there are several vendor-based approaches available, but application in routine cardiac SPECT is rare. This is partly as a result of the general attitude: 'in absence of attenuation correction, why bother with anything else!' The vendor-supplied solutions for cardiac SPECT motion correction tend to be limited and so, with exception of sites that undertake their own development, there is limited opportunity to fully address the effects of motion, especially during dynamic acquisition.

The results of Wang et al. ${ }^{1}$ confirm earlier work that demonstrated the importance of quantification. For example, Wells et al. ${ }^{6}$ have used a dedicated cardiac camera with CZT detectors and CT was performed separately for absolute quantification of MBF in an ischemic pig model using attenuation and scatter-corrected dynamic SPECT imaging. Attenuation and scatter correction improved the accuracy of the images while increasing noise, with an overall good correlation with MBF measured by microspheres. It is somewhat obvious that the estimation of absolute flow requires accurate quantification, but it might be expected that the necessary corrections are independent of stress or rest acquisition, especially as the SPECT acquisition, unlike PET, takes place at some time following stress. In the reported study, there was some evidence that this was not the case, suggesting that lack of correction could result in some difference between stress and rest studies, especially in ischemic zones, which consequently could affect estimation of myocardial flow reserve (MFR).
This was not statistically proven, but it does raise questions as to the validity of earlier work where MFR was estimated without any correction, on the basis that errors would be expected to equally affect both rest and stress MBF values and so cancel in the case of MFR. Despite these findings, in a recent study, in 95 patients there was a good correlation between MFR obtained from dynamic SPECT and total perfusion deficit using a dedicated cardiac camera with CZT detectors with no correction for attenuation. In addition, there was a good correlation between MFR and obstructive angiographic findings in 20 vessels and a stepwise reduction of MFR with increasing extent of obstructive CAD. ${ }^{7}$ It can be expected that the precision in estimating MFR could be large due to propagation of the errors in rest and stress MBF. The authors attempted to justify their findings, but the reason for systematic bias in MBF values remains unclear. ${ }^{1}$ This should be further evaluated in a larger population and verified in multiple centers.

In their study, Wang et al. ${ }^{1}$ utilized a fast rotation speed of up to 10 secs/rotation on a standard dual head camera (Siemens e-cam) to achieve sufficient temporal sampling for early frames following tracer administration and each acquisition was reconstructed assuming no temporal change during the short acquisition. Reconstruction of short acquisition frames can be challenging as there can be bias in low-count frames, especially if scatter correction is performed. Similar or shorter acquisition has been demonstrated to be feasible using the D-SPECT system (Spectrum Dynamics) where full rotation of bulky detectors is avoided, ${ }^{7}$ whereas continuous acquisition is available on the stationary multipinhole system of GE Healthcare, ${ }^{8}$ so there is no limit to the temporal sampling in this case. Ultra-fast acquisition might not be possible with all dual head systems. However, an appealing alternative is to perform 4D reconstruction that accounts for the rotation during acquisition, reducing the necessity for ultra-short rotation and facilitating dynamic acquisition for most standard dual head cameras. ${ }^{9}$

There is an increasing emphasis on quantification using SPECT and SPECT/CT ${ }^{10}$ and increasing evidence that reasonable accuracy can be achieved, ${ }^{11,12}$ at least for oncology, provided that care is taken to account for the various contributing factors. For the heart, there has been a reliance on relative perfusion for many years and the challenges in achieving full quantification are formidable. The paper by Wang et al. states the obvious in pointing out the need for full correction of dynamic data if accurate parameter estimation is sought. It further emphasizes the need for the community to at least adopt attenuation correction as a standard of practice, with a clear message to vendors that provision for this option should be mandatory. Most vendors support scatter 
correction and resolution modeling, although implementation differences across vendors can complicate the standardization of procedures. There is definitely a need for more rigorous attention to partial volume correction and motion correction, with translation to SPECT of procedures that are becoming available to the PET community.

\section{Acknowledgment}

Dr Brian Hutton receives support from the UK National Institute of Health Research University College London Hospitals Biomedical Research Centre.

\section{References}

1. Wang L, Wu D, Yang Y, Chen I-J, Lin C-Y, Hsu B, et al. Avoiding full corrections in Dynamic SPECT images impacts the performance of SPECT myocardial perfusion blood flow quantitation. J Nucl Cardiol 2016;. doi:10.1007/s12350-016-0513-4.

2. Hendel RC, Corbett JR, Cullom SJ, Depuey EG, Garcia EV, Bateman TM. The value and practice of attenuation correction for myocardial perfusion spect imaging: A joint position statement from the American Society of Nuclear Cardiology and the Society of Nuclear Medicine. J Nucl Med 2002;43:273-80.

3. Erlandsson K, Buvat I, Pretorius PH, Thomas BA, Hutton BF. A review of partial volume correction techniques for emission tomography and their applications in neurology, cardiology and oncology. Phys Med Biol 2012;57:R119-59.
4. Schafers KP, Stegger L. Combined imaging of molecular function and morphology: Image fusion and motion correction. Basic Res Cardiol 2008;103:191-9.

5. Gullberg GT, Reutter BW, Sitek A, Maltz JS, Budinger TF. Dynamic single photon emission computed tomography-Basic principles and cardiac applications. Phys Med Biol 2010;55:R11191.

6. Wells RG, Timmins R, Klein R, Lockwood J, Marvin B, deKemp RA, et al. Dynamic SPECT measurement of absolute myocardial blood flow in a porcine model. J Nucl Med 2014;55:1-7.

7. Ben-Haim S, Murthy VL, Breault C, Allie R, Sitek A, Roth N, et al. Quantification of myocardial perfusion reserve using dynamic SPECT imaging in humans: A feasibility study. J Nucl Med 2013;54:873-9.

8. Bocher M, Blevis IM, Tsukerman L. Shrem Y, Kovaski G, Volokh L. A fast cardiac gamma camera with dynamic SPECT capabilities: design, system validation and future potential. Eur J Nucl Med Mol Imaging 2010;37:1887-902.

9. Shrestha U, Sciammarella M, Alhassen F, Yeghiazarians Y, Ellin $\mathrm{J}$, Verdin E, et al. Measurement of absolute myocardial blood flow in humans using dynamic cardiac SPECT and 99mTc-Tetrofosmin: method and validation. J Nucl Cardiol 2015; doi: 10.1007/s12350-015-0320-3.

10. Ritt P, Vija H, Hornegger J, Kuwert T. Absolute quantification in SPECT. Eur J Nucl Med Mol Imaging 2011;38:S69-77.

11. Willowson K, Bailey DL, Baldock C. Quantitative SPECT reconstruction using CT-derived corrections. Phys Med Biol 2008;53:3099-112.

12. Shcherbinin S, Celler A, Belhocine T, Vanderwerf R, Driedger A. Accuracy of quantitative reconstructions in SPECT/CT imaging. Phys Med Biol 2008;53:4595-604 